CORRECTION

https://doi.org/10.1038/s41586-019-1209-0

\title{
Publisher Correction: Structural basis of ligand recognition at the human $\mathrm{MT}_{1}$ melatonin receptor
}

Benjamin Stauch, Linda C. Johansson, John D. McCorvy, Nilkanth Patel, Gye Won Han, Xi-Ping Huang, Cornelius Gati, Alexander Batyuk, Samuel T. Slocum, Andrii Ishchenko, Wolfgang Brehm, Thomas A. White, Nairie Michaelian, Caleb Madsen, Lan Zhu, Thomas D. Grant, Jessica M. Grandner, Anna Shiriaeva, Reid H. J. Olsen, Alexandra R. Tribo, Saïd Yous, Raymond C. Stevens,

Uwe Weierstall, Vsevolod Katritch, Bryan L. Roth, Wei Liu \& Vadim Cherezov

Correction to: Nature https://doi.org/10.1038/s41586-019-1141-3, published online 24 April 2019.

In Fig. 1 of this Letter, owing to an error in the production process, the rotation signs around $90^{\circ}, 135^{\circ}$ and $15^{\circ}$ were inadvertently missing. In addition, in the HTML version of the Letter, Extended Data Tables 2 and 3 showed the wrong tables (these were correct in the PDF). These errors have been corrected online. 